\title{
PROLONGED SURVIVAL BUT ULTIMATE CELL DEATH OF THE RAINBOW TROUT MACROPHAGE CELL LINE, RTS11, UNDER DIFFERENT STARVATION REGIMENS
}

\author{
Phuc H Pham ${ }^{1}$, Jun-Wen Li ${ }^{1}$, Dustin Ammendolia ${ }^{1}$, Patrick G Pumputis ${ }^{1}$, Lucy EJ \\ Lee $^{2}$, Niels C Bols ${ }^{1^{*}}$ \\ ${ }^{I}$ Department of Biology, University of Waterloo, Waterloo, ON, N2L 3Gl, Canada \\ ${ }^{2}$ Department of Biology, The University of the Fraser Valley, Abbotsford, BC, V2S 7M8, Canada
}

\begin{abstract}
Starvation is part of the natural life cycle of the salmonids and arises during several aquaculture practices. Occasionally starvation is even used to control infectious diseases, but how nutrient deprivation impacts the innate immune system is poorly understood, with both beneficial and detrimental effects being observed. Macrophages are the central cell type of the innate immune system and knowledge of their responses to starvation would help in understanding the influence of nutrient restriction on the innate immune system. Yet monitoring macrophage responses to starvation in vivo is difficult, especially to different kinds of nutrient deprivation. Therefore the rainbow trout monocyte-macrophage cell line, RTS11, was used as an in vitro alternative. RTS11 was routinely maintained in the basal medium, L-15, supplemented with $15 \%$ fetal bovine serum (FBS), which served as the control medium. The macrophages were subjected to three kinds of starvation. Serum starvation was done in L15 alone; amino acid and vitamin starvation was done in L15/ex, which has galactose and pyruvate; and extreme starvation was done in L15/salts, which has no nutrients. As along as initial cell density was high, RTS11 cultures could be maintained with no signs of cell death for at least 30 days in serum-free medium, and 14 days in L15/ex. By contrast, during extreme starvation multiple signs of apoptosis were seen between 10 and 14 days. These include an increase in caspase 3/7 activity, phosphatidyl serine externalization, and DNA laddering. Yet despite this, some cells in cultures with L15/salts continue to survive for at least 14 days. Overall RTS11 has been found to have a remarkable ability to withstand starvation and how specific macrophage activities are impacted by nutrient deprivation can be studied.
\end{abstract}

\section{KEYWORDS}

macrophages, RTS11, innate immunity, starvation

*Corresponding author. Tel: 1-519-888-4567- 33993; Fax: 1-519-746-4064

E-mail address: ncbols@uwaterloo,ca 